Methods This work presents findings from the formative evaluation, which utilised in-depth interviews, service data analysis and time-log methods.

Results All new patients have been contacted by the coordinator within two weeks of receipt of referral, with the aim of establishing themselves as their single point of access.

- The ability to have more frequent caseload review has allowed for the identification of care needs in a timelier way

- Conversations about care for people with MND can be emotive. Due to their clinical experience as a senior nurse, the coordinator has provided appropriate psychological support in conjunction with coordinating care

- The coordinator co-organised a professionals MND study day, as well as completing informal MND training to hospice staff and has made improvements to the MultiDisciplinary Team clinics to encourage a person-centred approach.

Conclusion Evidence to date suggests structures have been put in place to establish the coordinator as a single point of access locally, as well as to support patients, families and other professionals alike. Future evaluation will assess the extent this may have had an impact for people living with MND and their families.

\section{P-177 EVALUATION OF JOINT WORKING BETWEEN THE RENAL AND PALLIATIVE CARE TEAMS IN A HOSPITAL AT EAST LONDON}

\footnotetext{
${ }^{1}$ Ngai Mun Aiman Entwistle, ${ }^{2}$ Andrea Cove-Smith, ${ }^{3}$ Margaret Clifford, ${ }^{2}$ Suzanne Forbes, ${ }^{2}$ Jaryn Go, ${ }^{2}$ Clare Phillips. ${ }^{1}$ Barts and the London School of Medicine and Dentistry, London, UK; ${ }^{2}$ Barts Health NHS Trust, London, UK; ${ }^{3}$ St Joseph's Hospice, London, UK
}

\subsection{6/bmjspcare-2018-hospiceabs.202}

Introduction Identifying dialysis patients who are deteriorating and require a palliative care approach is challenging. Few have advance care planning (ACP) discussions and the majority die in hospital (Elliott \& Gessert, 2016; Davison, 2010). Joint working between the dialysis team and the Specialist Palliative Care team (SPC) was introduced following the recommendations of the UK End of Life Care Strategy (NHS Kidney Care, 2009). Dialysis patients likely to be in the last year of life according to the Gold Standards Framework (GSF) prognostic criteria were added to a 'Cause for Concern' register and discussed at a monthly renal/palliative care Multi-Disciplinary Meeting (MDM) attended by the hospital and community SPC teams. We aim to evaluate the impact of renal/ SPC joint working.

Methods Firstly, we reviewed records of all dialysis patients three months before and 15 months after the joint working began. Secondly, we reviewed records of dialysis patients who died over a one-year-period before and after the introduction of the joint working. Statistical tests were performed to compare the situation pre- and post- introduction of the joint working. Thirdly, semi-structured interviews were conducted with renal staff and the data analysed using thematic analysis. Results The proportion of dialysis patients being offered an ACP discussion increased by five-fold $(p=8.4 \mathrm{E}-04)$, ACP discussion documentation increased from zero to $3.5 \%(p=3.7 \mathrm{E}$ 03 ) and proportion with Coordinate My Care (CMC) increased from zero to $3.14 \% \quad(p=7.4 \mathrm{E}-03)$. The staff interviewed $(n=8)$ highlighted the benefits of working alongside the SPC team, especially on symptom management and community support.

Conclusion Introduction of the joint working appears to have improved ACP and delivery of palliative care to dialysis patients. It is hoped that further embedding routine screening with GSF prognostic criteria, MDM discussions and renal-SPC collaboration will continue to improve palliative care for dialysis patients.

\section{P-178 COST-EFFECTIVE INNOVATIONS FOR INTEGRATION OF PALLIATIVE CARE AND RESPIRATORY SERVICES}

${ }^{1}$ Claire Ferguson, ${ }^{2}$ Geraldine Burge, ${ }^{2}$ Sue Barclay. 'Marie Curie Hospice, West Midlands, Solihull, UK; ${ }^{2}$ University Hospitals Birmingham NHS Foundation Trust, Birmingham, UK

\subsection{6/bmjspcare-2018-hospiceabs.203}

Background It is recognised that chronic respiratory patients benefit from palliative care due to their burden of symptoms and risk of inappropriate medical interventions at end of life (Joshi, Joshi, \& Bartter, 2012; Taylor \& Murray, 2018). However, respiratory referrals to our palliative care services have remained low. Research has shown that patients and staff can equate palliative care with 'giving up' (Smallwood, Gaffrey, Gorelik et al., 2018) when exacerbations are potentially reversible, resulting in patients dying in hospital, whilst undergoing active medical management.

Aims Understand the needs of chronic respiratory patients. Increase respiratory referrals to hospice services. Increase knowledge and skills for healthcare professionals in managing palliative respiratory patients.

Methods Using contacts from the community specialist nurses and hospital palliative care team, a quarterly meeting at the hospice was set up for interested healthcare professionals from the hospice, community and hospital involved in working with chronic respiratory patients. By sharing information on the services available and discussing complex respiratory cases, new ways of working together were identified. These included:

- Respiratory nurses observing consultant ward rounds at the hospice

- The hospital oxygen clinic being held at the hospice

- The Interstitial Lung Disease support group moving to the hospice

- Developing a respiratory breakfast club at the hospital for palliative and respiratory staff to discuss the management of hospital and community respiratory patients

- Hosting a regional conference on interstitial lung disease at the hospice

- Joint home visits with the respiratory and palliative care teams.

Results Anecdotal evidence so far has shown an increase in referrals of respiratory patients to Hospice services and these patients have benefitted from palliative care. Quantifiable data will be available by November 2018 .

Conclusions It is possible to increase referrals from respiratory to palliative care without expensive or time-consuming interventions. Developing relationships and improving understanding between the teams has helped to break down barriers and led to further collaborative working. 